\title{
Effects of Voluntary Running Exercise on Blood Pressure and Renin-Angiotensin System in Spontaneously Hypertensive Rats and Normotensive Wistar-Kyoto Rats
}

\author{
Atsumi Hayashi, Ai Kobayashi, Rumiko TaKaHashi, Fumiaki Suzuki, ${ }^{1}$ \\ Tora NAKAGAWA ${ }^{1}$ and Koichi KiмOTO \\ Department of Nutrition, Tokyo Kasei University, Itabashi-ku, Tokyo 173-8602, Japan \\ ${ }^{1}$ Center of Gene Biotechnology, Faculty of Agriculture, Gifu University, Gifu 501-1112, Japan
}

(Received August 9, 1999)

\begin{abstract}
Summary The effect of a voluntary running exercise on blood pressure and renin-angiotensin system (RAS) was studied in male spontaneously hypertensive rats (SHR) and normotensive Wistar-Kyoto rats (WKY). SHR and WKY were assigned to either voluntary running exercise or sedentary control groups at $5 \mathrm{wk}$ of age. The systolic blood pressure in the exercised group for both strains of rats was significantly lower than in the sedentary control group. The plasma renin activity (PRA) and plasma renin concentration (PRC) were significantly lower in voluntary running exercised SHR than in sedentary SHR, whereas the same exercise did not result in a lower PRA and PRC in WKY. These results suggested that the blood pressure lowering effect of voluntary running exercise is related to the suppression of RAS in SHR.
\end{abstract}

Key Words voluntary running exercise, blood pressure, PRA, PRC

Hypertension is a life-style related diseases, and the first treatment begins with the improvement of one's life-style. After that, in many cases, medical therapy is used along with nonmedical therapy. Antihypertensive drugs, however, produce various kinds of side effects (1). The nonmedical therapies are composed of limiting meals, exercise, limiting alcohol, and other things. Several lines of evidence from animal studies and clinical observations strengthen the view that exercise training lowers blood pressure in hypertensive animals $(2,3)$ and humans $(4)$. The antihypertensive mechanism because of exercise is reported to be associated with the decrease in body fluid (5), in the cardiac output derived from a decreased heart rate (6), in peripheral resistance (7), and in the sympathetic nervous activity (8). It is not yet clear, however, whether the RAS can produce antihypertensive effects by exercise.

As for the studies of exercise training with a small animal, running on a treadmill or swimming was often used. These methods are done by forcibly loading exercise against the small animal, and the following results have been reported: the blood pressure can be decreased or be elevated. The voluntary running exercise is called gentle impatience training compared with those exercise loadings with stress $(9,10)$. The forced exercise was not chosen as the method for this experiment because the stress might effect the blood pressure (11) and renin activity (12). We therefore chose voluntary running exercise as a nonstress experiment and investigated the relationship between blood pressure and renin activity.

\section{MATERIALS AND METHODS}

Experimental animals and rearing methods. Seventeen SHR and 16 WKY rats were obtained from Charles
River Japan Inc. The male rats were divided into two groups at 5 wk of age. One group (8 SHR and 8 WKY rats) was housed in individual wheel cages with $1 \mathrm{~m}$ circumference units, which allowed free access to a voluntary wheel running for $11 \mathrm{wk}$, and the other group (9 SHR and 8 WKY rats) was kept under individual sedentary conditions. The rats were raised in a room with the temperature set at $23 \pm 1{ }^{\circ} \mathrm{C}$, the humidity at $55 \pm 5 \%$ and lighting for $12 \mathrm{~h}$ daily (lighted time from 6:00 to 18:00). The diet (CE-2; Na 0.39\%, K 0.98\%, CLEA Japan, Inc., Tokyo) and drinking water were given ad libitum.

Analytical procedures. During the experimental period, body weights, food intakes, and running distance were measured every day. Systolic blood pressure was measured once a week without anesthesia by the tailcuff method (BP 98A Softron, Inc., Tokyo). Before the end of the experiment, a blood sample with EDTA was obtained from the caudal vein for assays of PRA and PRC and centrifuged to separate the plasma, which was stored at $-80^{\circ} \mathrm{C}$ until analysis.

Assays of PRA and PRC. The PRA and PRC were measured by a modified method (13), using the enzyme linked immunosorbent assay (ELISA) of Suzuki et al. (14). Rat plasma was used for an assay of PRA. Rat plasma and recombinant angiotensinogen (15) as substrate were used for an assay of PRC.

The detection was measured by a chemiluminescence enzyme immunoassay (CLEIA) method (13) with luminol, using ML-3000 (Microtiter plate luminometer, Dynatech Lab., USA). A standard curve in the plot with BioLinx was done, and the AI quantity in the plasma was calculated. It showed the renin activated value for the AI weight at which renin in $1 \mathrm{~mL}$ of plasma gener- 

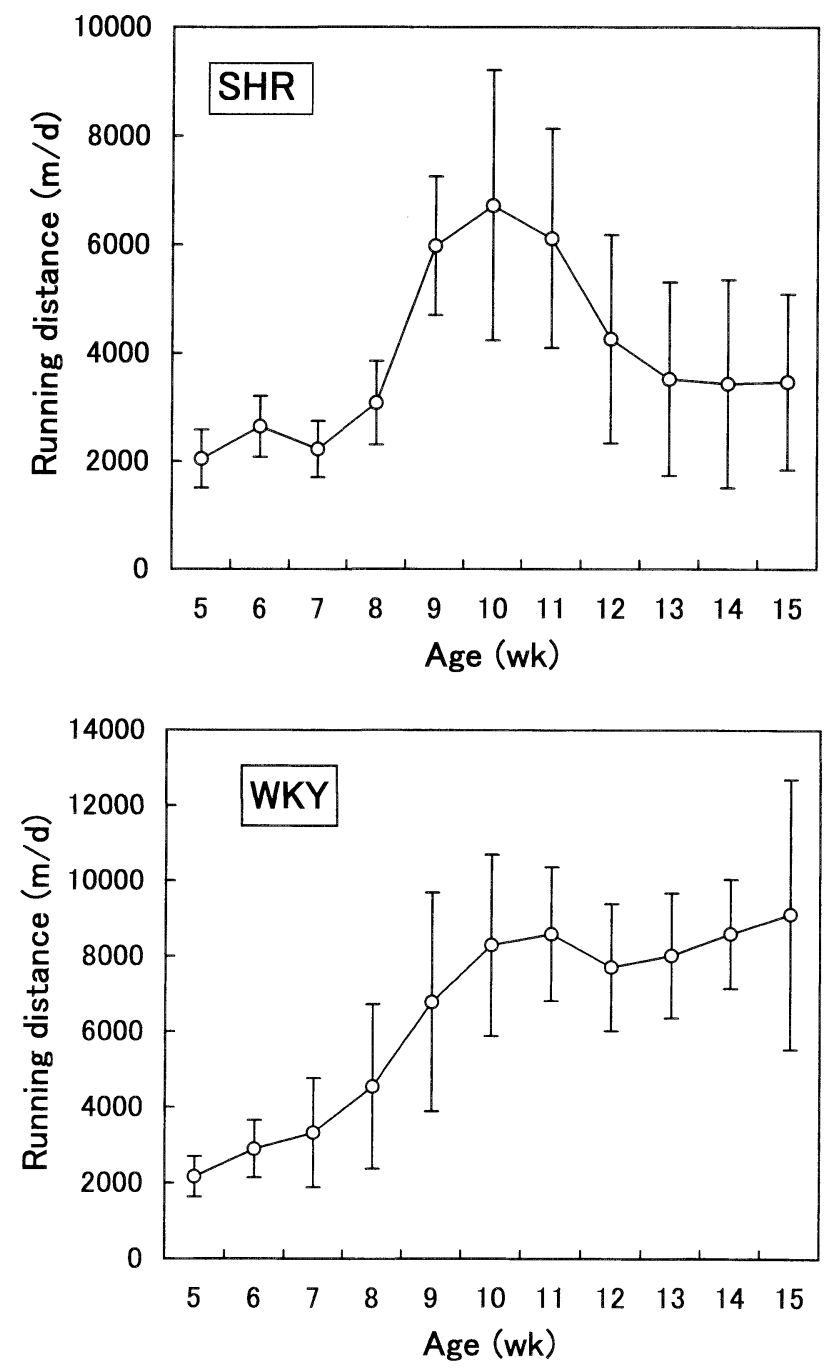

Fig. 1. Daily running distance of exercised group in SHR and WKY. The values are expressed as mean \pm SD.

ates in $1 \mathrm{~h}$.

Statistical analysis. Data were expressed as mean \pm SD. The significance of differences was assessed by a Student's $t$-test or Welch's $t$-test. The selected significance level was $p<0.05$.

\section{RESULTS}

\section{Running distance}

Figure 1 shows the running distance $(\mathrm{m} / \mathrm{d})$ of the exercised groups. The running distance of SHR increased from $10 \mathrm{wk}$ of age to $6,720 \mathrm{~m} / \mathrm{d}$, then declined to $3,466 \mathrm{~m} / \mathrm{d}$ by $15 \mathrm{wk}$ of age. In the case of WKY, however it was prolonged approximately to $8 \mathrm{~km} / \mathrm{d}$ at $10 \mathrm{wk}$ of age, and it was maintained to the end of experimental period at $15 \mathrm{wk}$ of age.

Food intake and body weight

As shown in Fig. 2, food intakes of the exercised groups were significantly higher than the control groups for both the SHR and WKY cases. Their body weights are shown in Fig. 3. The SHR body weight was significantly increased in the exercised group. The body weight was not different, however, between exercise or control groups in WKY.
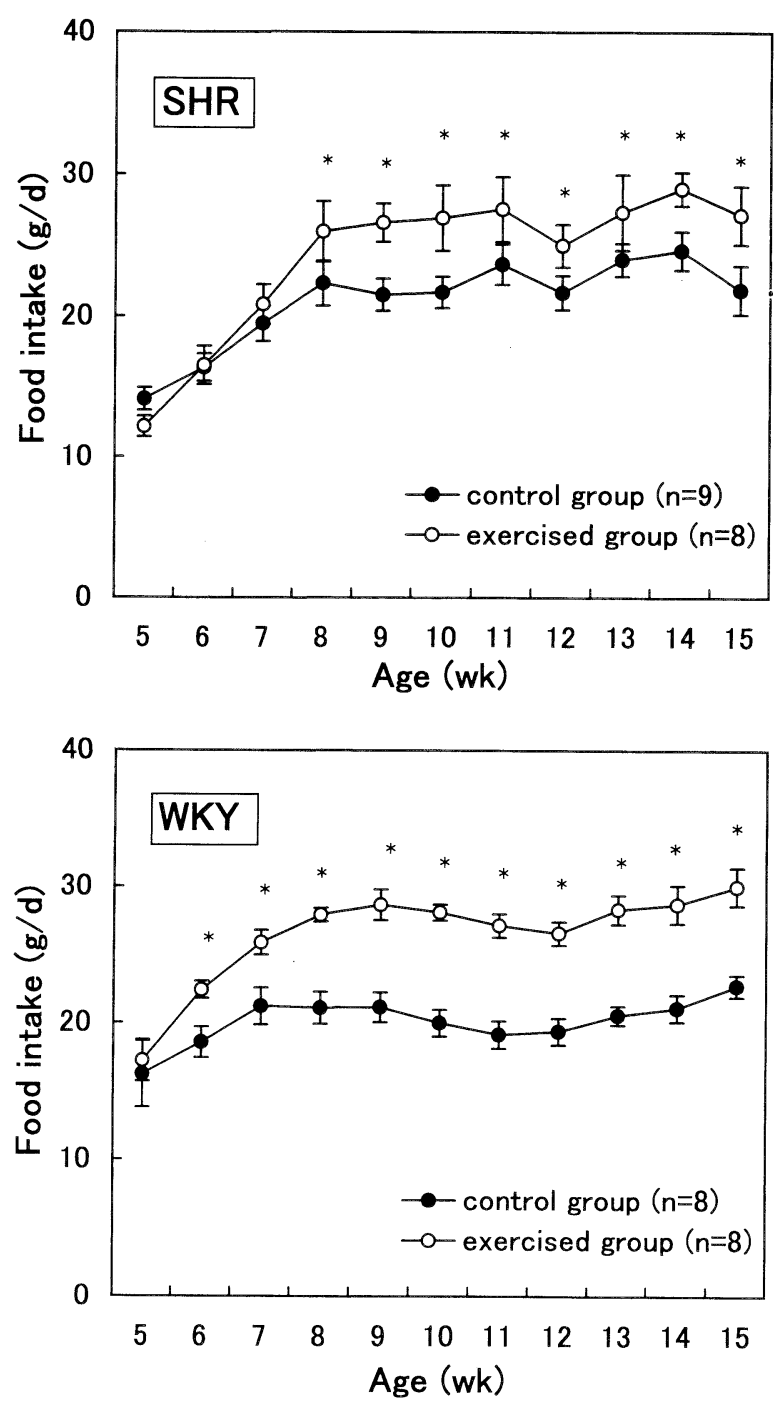

Fig. 2. Food intake of exercised group and control group in SHR and WKY. - , control group; $\bigcirc$, exercised group. The values are expressed as mean \pm SD. ${ }^{*} p<0.05$, compared with the control group.

\section{Systolic blood pressure}

The change in the systolic blood pressure during the experimental period is shown in Fig. 4. Since 8-9 wk of age, the systolic blood pressure in the exercised groups of both rats was significantly decreased compared with both control groups. At the end of the experimental period at $15 \mathrm{wk}$ of age, systolic blood pressure was $204 \pm 8 \mathrm{mmHg}$, and $172 \pm 14 \mathrm{mmHg}$ in sedentary SHR and exercise SHR, respectively. The increase in blood pressure in SHR was extremely inhibited by the voluntary running exercise. In WKY, the systolic blood pressure significantly decreased in the exercised group $(126 \pm 7 \mathrm{mmHg}$ ) compared with that in the control group (134 $5 \mathrm{mmHg}$ ).

$P R A$ and $P R C$

As shown in Fig. 5, in SHR, PRA was significantly decreased in the exercised group. PRC in the exercised group was also significantly lower than in the control group. In WKY, however, no difference was observed between the exercised group and the control group.

In the nonexercised group of SHR, a significant nega- 

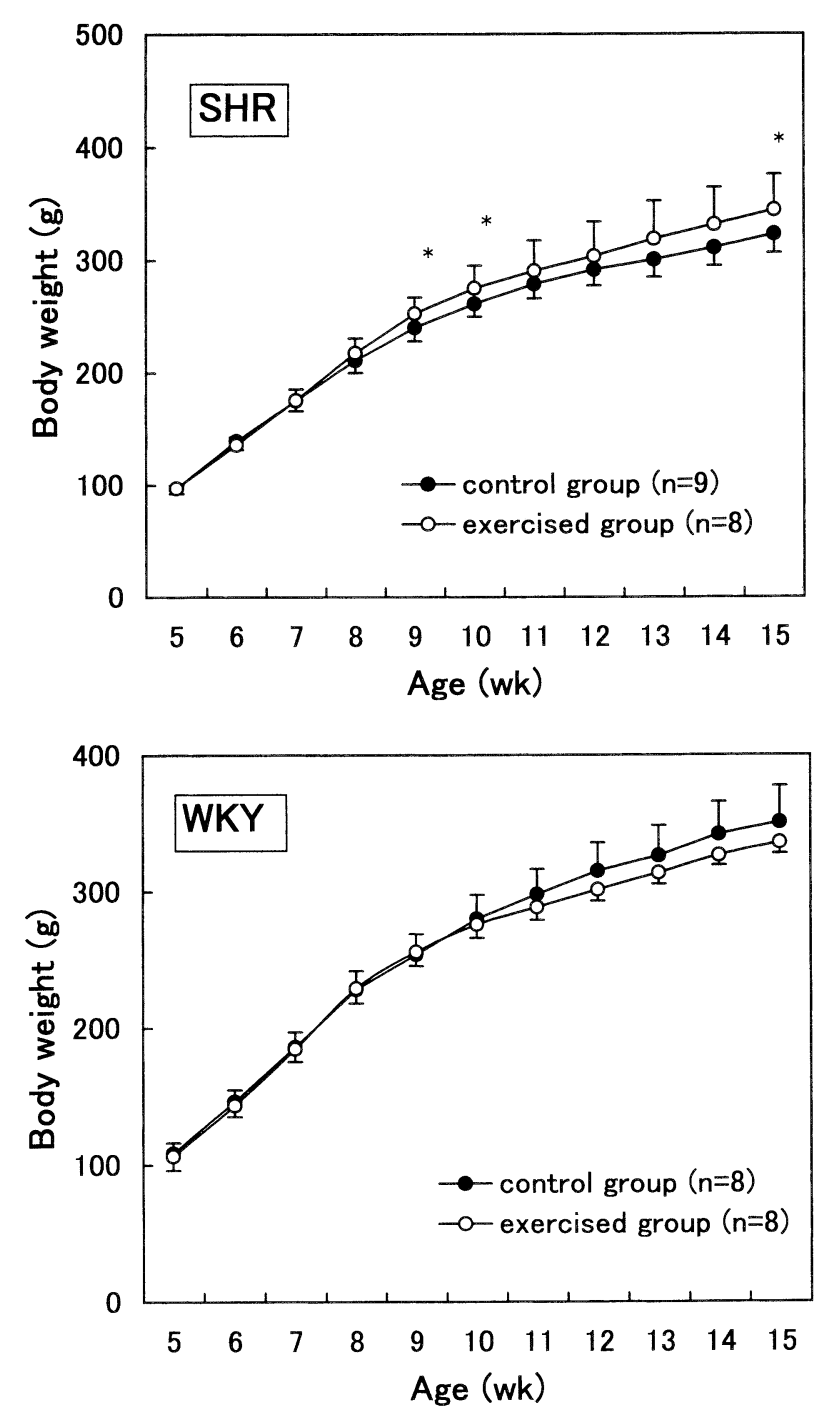

Fig. 3. Effects of exercise on the body weight of SHR and WKY. - control group; $\bigcirc$, exercised group. The values are expressed as mean $\pm \mathrm{SD}$. ${ }^{*} p<0.05$, compared with the control group.

tive correlation was observed between the PRA and blood pressure and between PRC and blood pressure. In the exercised group of SHR, a weak positive correlation was observed (Fig. 6a, b). In the nonexercised group of WKY, no correlation was observed between the PRA and blood pressure and between PRC and blood pressure. In the exercised group of WKY, however a significant positive correlation was observed between PRA and blood pressure, but weak negative correlation was observed between PRC and blood pressure (Fig. 6c, d). Thus in WKY, the influence of the voluntary running exercise was different in the case of SHR.

\section{DISCUSSION}

In recent years, antihypertensive exercise therapy is becoming important based on many studies (16). In 1993, the exercise therapy was positively adopted into the guidelines for the treatment of mild hypertension by the World Health Organization-International Society of Hypertension (17). We investigated the effect of voluntary running exercise on the blood pressure and renin
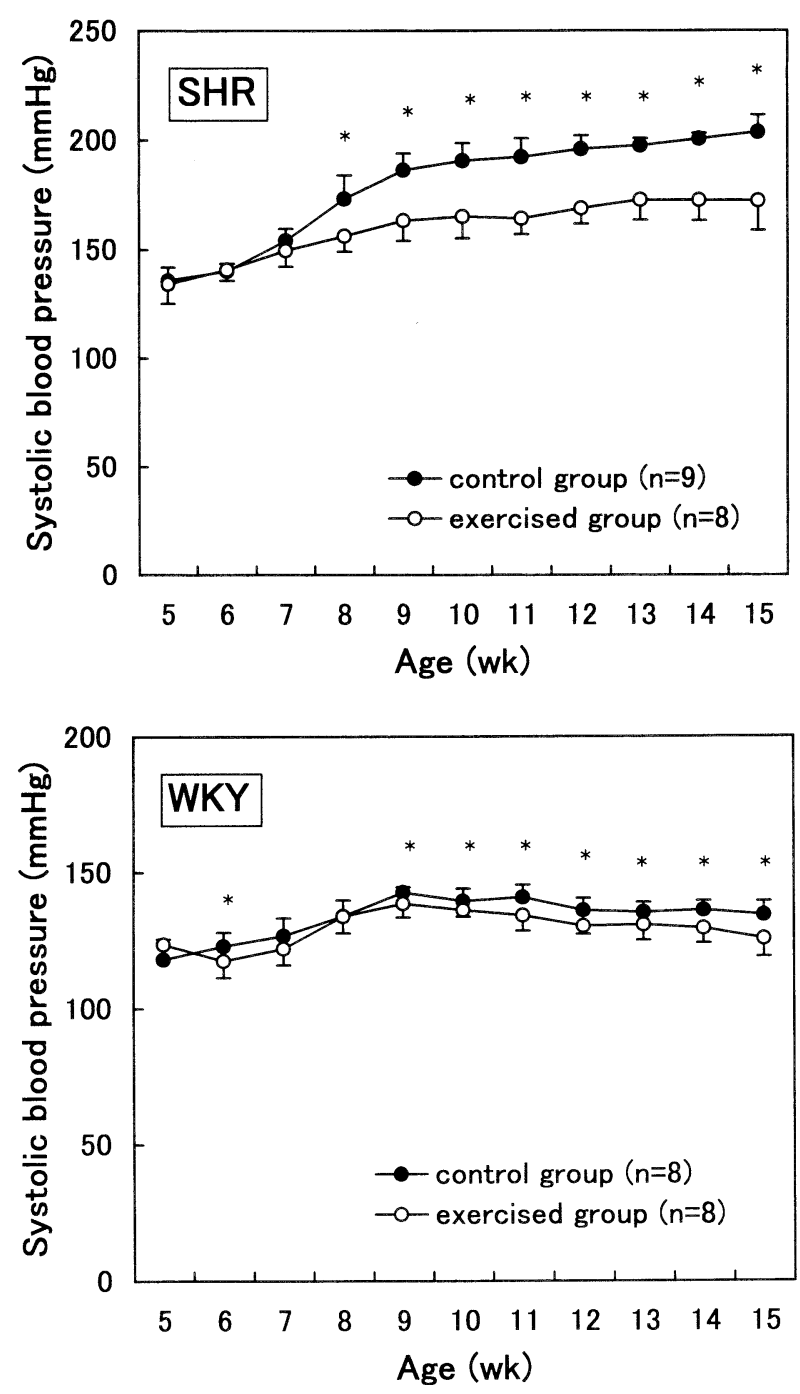

Fig. 4. Effects of exercise on blood pressure of SHR and WKY. - control group; $\bigcirc$, exercised group. The values are expressed as mean \pm SD. ${ }^{*} p<0.05$, compared with the control group.

activity. We feared that the stress by loading forced exercise might influence the blood pressure, the renin activity, and the sympathetic nervous system. We therefore chose the voluntary running exercise to exclude the stress.

The food intake of SHR and WKY was significantly increased by the voluntary running exercise. In SHR, the increase in the body weight of the exercised group was also significantly higher than in the control group. This result was similar to the report by Oshima and Suzuki (2). In the case of WKY, however, the body weight of the exercised group was not higher than the control group. It is generally known that breeding with a diet ad libitum in the narrow cage may result in an excessive body weight for rats. Therefore it is speculated that the excessive body weight could be improved by exercise. SHR is disease-bearing animals, usually small, and has a light body weight compared with WKY. Therefore the improvement in the physiological conditions, such as the blood pressure, food intake, and body weight, might occur in SHR because of exercise. Kingwell 

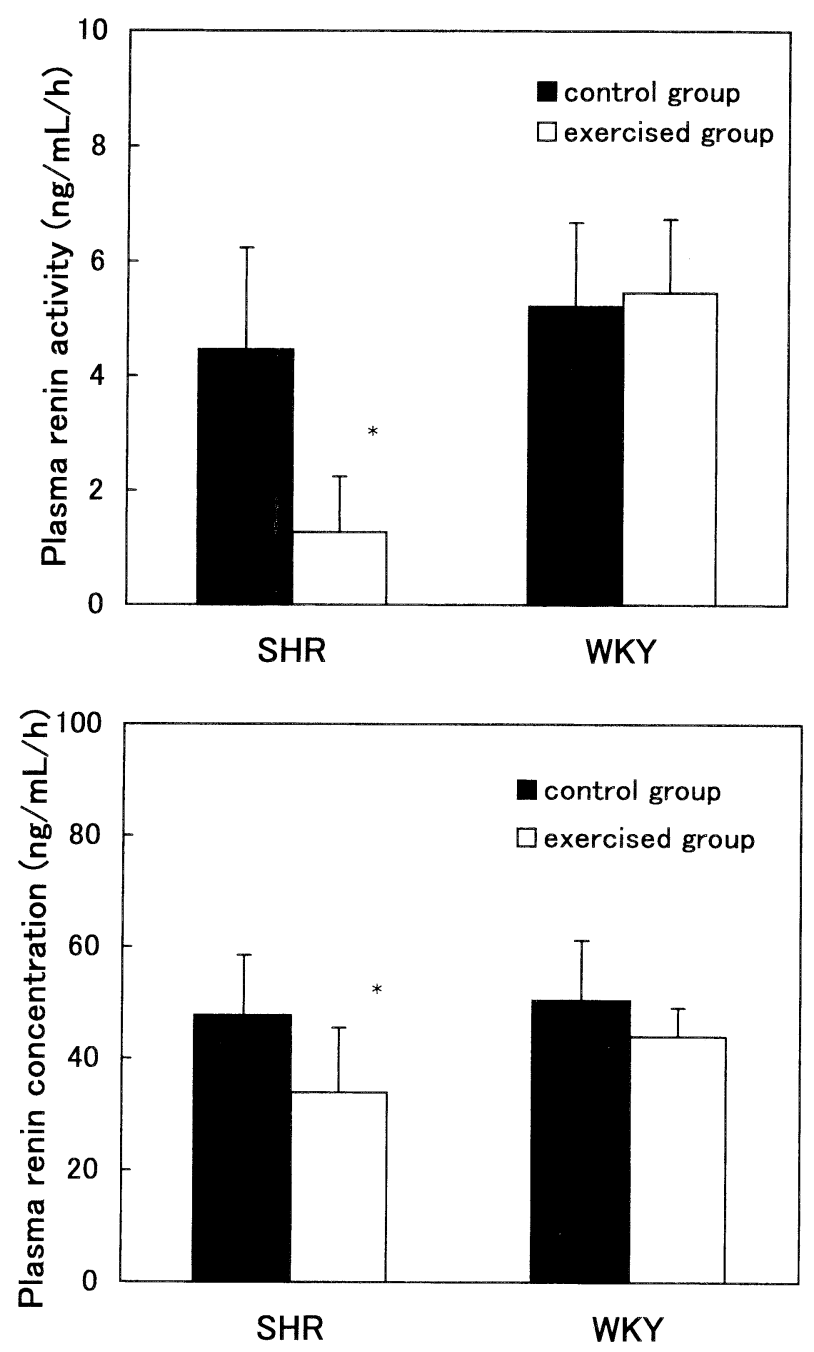

Fig. 5. Effects of exercise on plasma renin activity and plasma renin concentration in SHR and WKY. $\mathbf{\square}$, control group; $\square$, exercised group. The values are expressed as mean $\pm \mathrm{SD} .{ }^{*} p<0.05$, compared with control group.

et al. (18), however, reported that in normotensive WKY, the aortic compliance increased after the exercise, but no increase in SHR was observed. On the other hand, Suzuki et al. (3) showed that there was a depressor effect against the obesity under the condition of relatively light exercise (voluntary running exercise) in the normotensive rat. This phenomenon was not observed, however, with the intense exercise of more than $5 \mathrm{~km}$.

Hashimoto et al. (9) and Ito et al. (19) reported that the forced exercise did not depress the blood pressure of SHR, but the long-term exercise (voluntary running exercise) depressed the blood pressure. In this study, the voluntary running exercise significantly decreased the blood pressure, not only in SHR, but also in WKY. It was also found that a reduction in blood pressure by the exercise was not characteristic for only the hypertensive rat. This result agreed with the report by Suzuki et al. (3). According to the study of Oshima and Suzuki (2), the first of $10 \mathrm{wk}$ of SHR is a rapid growth period, which showed a significant positive correlation between the running distance (on the voluntary running exercise) and the blood pressure. But after $15 \mathrm{wk}$ of age, the growth was approximately complete; a significant negative correlation between the running distance and the blood pressure was then observed. Moreover, Kingwell et al. (18) suggested that SHR had the lower running activity (being $1.0 \pm 0.2 \mathrm{~km} / 24 \mathrm{~h}$ for $19-20 \mathrm{wk}$ of age) for the voluntary running exercise, which participated in the hypertension.

Because of the voluntary running exercise of SHR, the blood pressure was conspicuously depressed. Moreover, PRA and PRC were also significantly decreased in SHR. Consequently, in the case of SHR, it was suggested that the mechanisms of blood pressure reduction because of exercise might be a result of depression in the RAS. Development of hypertension in SHR, however, is the result of RAS, the sympathetic nervous system, the renal factor, and so on. There are various kinds of reports about the renin activity of SHR; some have reported high values $(20)$, but almost all showed a normal or low value (21-23). It was speculated that there was little possibility of RAS participation in the development of hypertension in SHR. There is no report, however, that RAS is directly related to the blood pressure decrease mechanism with exercise therapy. In the report of Ghaemmaghami et al. (24), the renin activity in SHR was not changed with swimming training. Hagberg et al. (6) reported a decrease in renin activity, and Matsusaki et al. (25) saw no change in renin activity before and after exercise therapy for essential hypertension patients. Therefore we investigated the effect of RAS on the blood pressure reduction. As a result, it was observed that PRA and PRC in SHR were significantly decreased by voluntary running exercise. Moreover, a significant negative correlation was observed between the blood pressure and PRA and blood pressure and PRC in the nonexercised group of SHR. A weak positive correlation in the exercised group of SHR was also observed. In other words, the blood pressure control by RAS in SHR was evaluated for suppression of renin secretion against high blood pressure. This is a wellknown reason why SHR have a low renin activity. On the other hand, in the exercised group a weak positive correlation tendency appeared. In spite of the low renin activity in SHR, because of the voluntary running exercise the renin activity was more depressed. In SHR, RAS participated in the blood pressure control as a result of the voluntary running exercise. However, Kiyonaga et al. (8) reported that the RAS did not change by exercise in the hypertension patient, but the blood pressure was more suppressed in the person with a low PRA before the exercise.

In WKY, no significant difference was observed in the PRA and PRC between the exercised group and the nonexercised group. In the exercised group, a significant positive correlation was between PRA and blood pressure. In WKY, PRA might be participated with the suppression of blood pressure.

Arakawa (4) reported that a change of RAS was observed in healthy persons, but it was not seen in the hypertension patient. Our result of SHR, however, sug- 


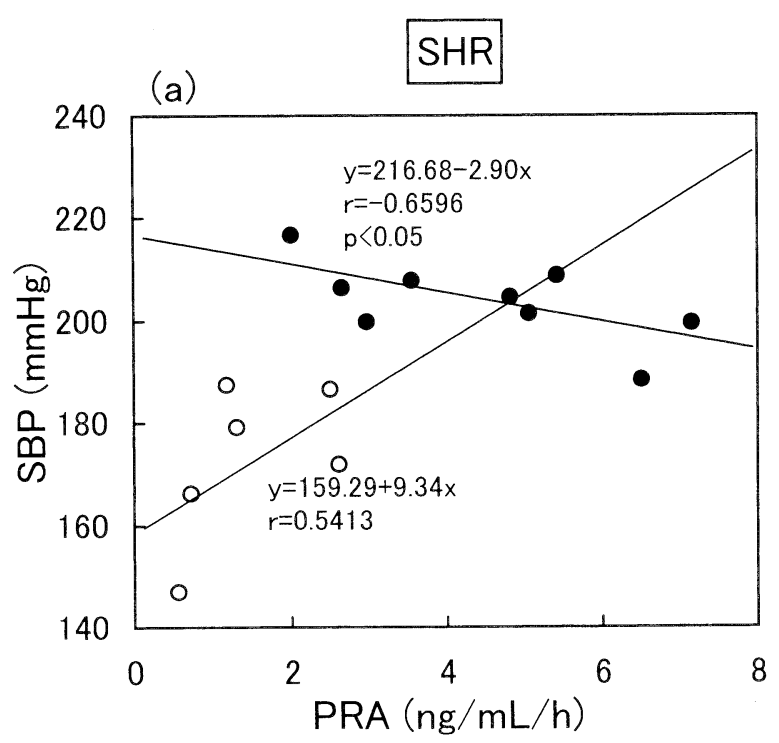

(b)

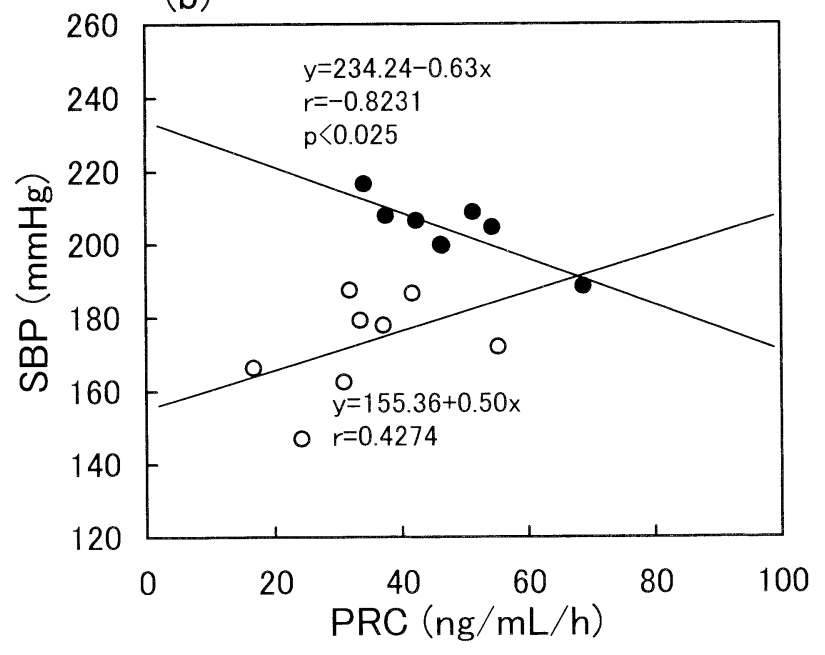

(c)

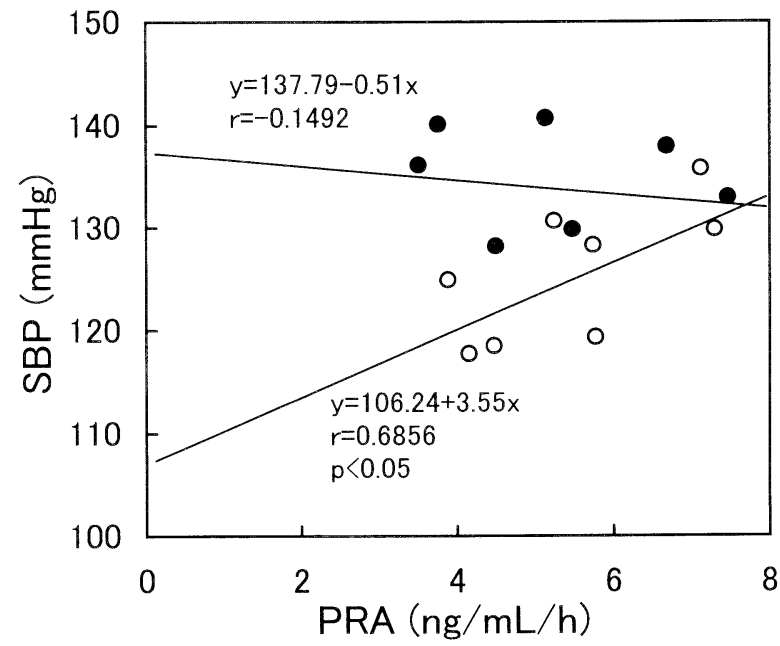

(d)

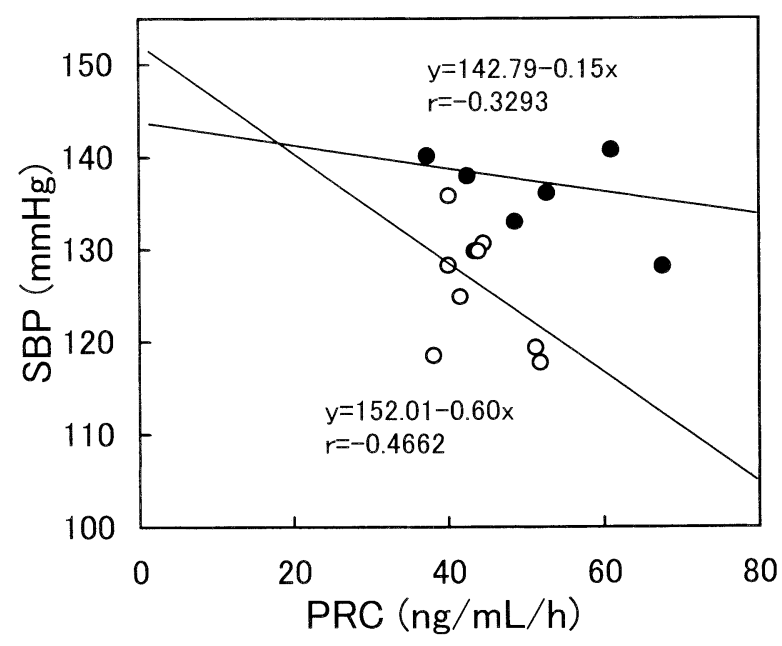

Fig. 6. Correlation between renin activity and systolic blood pressure in SHR and WKY: (a) correlation between PRA and SBP in SHR; (b) correlation between PRC and SBP in SHR; (c) correlation between PRA and SBP in WKY; (d) correlation between PRC and SBP in WKY. - control group; $\bigcirc$, exercised group. SBP, systolic blood pressure; PRA, plasma renin activity; PRC, plasma renin concentration.

gested that RAS had the possibility to participate in the suppression of blood pressure in the case of voluntary running exercise against low-renin hypertension. This leaves some problems for the adaptation of human hypertensive patients. In the case of low-renin hypertensions, it is difficult to find the effect of RAS, but with the voluntary running exercise, the role of RAS became apparent. From this experiment, it was then suggested that the function of RAS might appear in the case of voluntary running exercise. According to the report of Tuck et al. (26), the renin secretion was controlled under the sympathetic nervous system. Kiyonaga et al. (8) had reported that long-term exercise suppressed the sympathetic nervous systems. We plan to clear the relation of these systems for the control mechanism of the blood pressure.

\section{Acknowledgments}

The authors wish to express their appreciation to Ms. K. Taninaka, Ms. A. Sagara, Ms. K. Watabe, and Ms. C.
Shindo for their skillful technical assistance.

\section{REFERENCES}

1) Jachuck SJ, Brierley H, Jachuck S, Willcox PM. 1982. The effect of hypertensive drugs on the quality of life. $J R$ Coll Gen Pract 32: 103-105.

2) Oshima S, Suzuki S. 1976. Influence of physical exercise on blood pressure using the spontaneously hypertensive rat (SHR). Jpn J Nutr 34: 109-114.

3) Suzuki K, Machida K, Kariya M. 1992. Conditions for low-intensity voluntary wheel running in rats and its chronic effects on health indexes. Jpn J Hyg 47: 939-951.

4) Arakawa K. 1993. Hypertention and exercise. Clin Exp Hypertens 15: 1171-1179.

5) Melin B, Eclache JP, Geelen G, Annat G, Allevard AM, Jarsaillon E, Zebidi A, Legros JJ, Gharib Cl. 1980. Plasma AVP, neurophysin, renin activity, and aldosterone during submaximal exercise performed until exhaustion in trained and untrained men. Eur J Appl Physiol 44: 141-151. 
6) Hagberg JM, Montain SJ, Martin WH, Ehsani AA 1989. Effect of exercise training in 60- to 69-year-old persons with essential hypertension. Am J Cardiol 64 348-353.

7) Nelson L, Jennings GL, Esler MD, Korner P. 1986. Effect of changing level of physical activity on blood pressure and hemodynamics in essential hypertension. Lancet $\mathbf{2}$ : 473-476.

8) Kiyonaga A, Arakawa K, Tanaka H, Shindo M. 1985. Blood pressure and hormonal responses to aerobic exercise. Hypertension 7: 125-131.

9) Hashimoto I, Higuchi M, Yamakawa K, Suzuki S. 1981. Effect of chronic exercise on spontaneously hypertensive rats. J Phys Fitness Jpn 30: 206-213.

10) Higuchi M, Hashimoto I, Yamakawa K. 1982. Training effects of voluntary physical training. J Phys Fitness Jpn 31: 205-210.

11) Hashimoto I, Higuchi M, Yamada K, Suzuki S. 1981. Effect of exercise training on spontaneously hypertensive rats. Med Sci Sports Exer 13 (Suppl): 138.

12) Porter JP. 1990. Effect of stress on the control of renin release in spontaneously hypertensive rats. Hypertension 15: 310-317.

13) Kimoto K, Kuroda Y. 1996. Studies on renin and ACE activities of factors in hypertension. Bull Tokyo Kasei Univ 36: 53-58.

14) Suzuki F, Yamashita A, Takahashi A, Ito M, Miyazaki S, Nagata Y, Nakamura Y. 1990. Highly sensitive microplate-ELISA for angiotensin I using 3,3',5,5'tetramethylbenzidine. Clin Exp Hyper A1 2: 83-95.

15) Kimoto K, Iwai $N$, Higuchi $K$, Hatae $T$, Hori $H$, Fukamizu A, Murakami K, Inagami T. 1992. Expression and characterization of recombinant rat angiotensinogen derived from Chinese hamster ovary cells. Biomed Res 13: 41-46.

16) Arakawa K. 1993. Antihypertensive mechanism of exercise. J Hypertens 11: 223-229.

17) Guidelines Sub-Committee. 1993. Guidelines for the management of mild hypertension: Memorandum from a World Health Organization/International Society of Hypertension meeting. J Hypertens 11: 905-918.

18) Kingwell BA, Arnold PJ, Jennings GL, Dart AM. 1998. The effects of voluntary running on cardiac mass and aortic compliance in Wistar-Kyoto and spontaneously hypertensive rats. J Hypertens 16: 181-185.

19) Ito K, Takaishi T, Ito M, Takeuchi H, Hirofuji C. 1988. Effects of chronic exercise on blood pressure in spontaneously hypertensive rats. Bull Fac Educ Kobe Univ 81: 69-75.

20) De Jung W, Lovenberg W, Sjoerdsma A. 1972. Increased plasma renin activity in the spontaneously hypertensive rat. Proc Soc Exp Biol Med 139: 12131216.

21) Czyzewski LB, Pettinger WA. 1973. Failure of feedback suppression of renin release in the spontaneously hypertensive rat. Am J Physiol 225: 234-239.

22) Forman BH, Mulrow PJ. 1974. Effect of propranorol on blood pressure and plasma renin activity in the spontaneously hypertensive rat. Circ Res 35: 215-221.

23) Shiono K, Sokabe H. 1976. Renin-angiotensin system in spontaneously hypertensive rats. Am J Physiol 231 1295-1299.

24) Ghaemmaghami F, Allevard AM, Fareh J, Geelen G, Gharib C. 1991. Effects of acute exercise and prolonged exercise training on blood pressure, vasopressin and plasma renin activity in spontaneously hypertensive rats. Eur J Appl Physiol 62: 198-203.

25) Matsusaki M, Ikeda M, Tashiro E, Koga M, Miura S, Ideishi M, Tanaka H, Shindo M, Arakawa K. 1991. Influence of workload on the antihypertensive effect of exercise. Clin Exp Pharmacol Physiol 19: 471-479.

26) Tuck ML, Stern N, Sower JR. 1985. Enhanced $24 \mathrm{hr}$ norepinephrine and renin secretion in young patients with essential hypertension. Relation with the circadian pattern of arterial pressure. Am J Cardiol 55: 112-115. 